\title{
Somatic Mitochondrial Mutations in Oral Cavity Cancers among Senegalese Patients
}

\author{
Silly Toure ${ }^{1}$, Fatimata Mbaye ${ }^{2 *}$, Mame Diarra Gueye ${ }^{2}$, Malick Fall ${ }^{3}$, Ahmadou \\ Dem $^{4}$, Jean Baptiste Lamy ${ }^{5,6}$, Mbacké Sembene ${ }^{2}$
}

\begin{abstract}
Background: Somatic mutations affecting the mitochondrial DNA (mtDNA) have been frequently observed in human cancers and proposed as important oncological biomarkers. However, the exact mtDNA mutations that is responsible for the pathogenesis of cancer remains unclear. The aim of this study was to investigate somatic mutations in the MT-CYB and D-Loop regions of mitochondrial DNA (mtDNA) in oral cavity cancers from Senegalese patients.

Methods: MT-CYB and the D-Loop of mtDNA derived from 45 oral cavity cancer tissues and 21 control blood samples were assessed by PCR and sequencing. The sequences of MT-CYB and the D-Loop from cancerous tissues were compared with control sequences, and sequence differences were recognized as somatic mutations. Results: Overall, 389 somatic mtDNA mutations were identified, most of which $(79.43 \%)$ were located in the D-Loop region. The majority of base substitution mutations were G-to-A $(63.93 \%)$ and T-to-C $(16.39 \%)$ transitions. In the protein-coding MT-CYB gene, 29 missense mutations were observed. The pathogenic mutation load of MT-CYB was $3.11 \%$. Pathogenic mutations were carried by $25 \%$ of patients. pArg76Pro (pArg282Pro in rCRS) was novel and was the most common pathogenic mutation observed. Conclusion: These results strongly indicate that mtDNA mutations are a potential marker of oral cavity cancer.
\end{abstract}

Keywords: Oral cavity cancers- MT-CYB- D-Loop- mtDNA

Asian Pac J Cancer Prev, 20 (7), 2203-2208

\section{Introduction}

Oral cancers are malignant neoplasms occurring in the structures or tissues of the mouth. They are potentially fatal and constitute a considerable proportion of tumors of the head and neck. Regardless of the fact that the oral cavity is a prime target for the accumulation of carcinogens, most oral cancers are frequently detected at an advanced stage, leading to a lower survival rate among subjects. Oral cancers are currently a major global health issue: they are reported to be the sixth most common cancer in the world, with an estimated 400,000 newly diagnosed cases and causing 223,000 deaths in 2008 (Wamakulasuriya, 2009; Chaturvedi et al., 2013).

The majority of human cancer types are characterized by genetic instabilities. Mitochondrial DNA (mtDNA) is the only non-nuclear genetic component of the human genome (Stewart et al., 2008). Mitochondria are intracellular organelles responsible for ATP production via their oxidative phosphorylation system. It is widely accepted that mitochondria play an important role in the aging processes of both cells and individuals. Somatic mutations in mtDNA have been increasingly observed in human cancers and have been proposed as important oncological biomarkers (Jakupciak et al., 2005; Chatterjee et al., 2006). The biological significance of mtDNA mutations in cancer remains unclear (Chatterjee et al., 2006). Nevertheless, evidence is emerging that mtDNA damage has direct effects mediated by reactive oxygen species (Petros et al., 2005; Ishikawa et al., 2008).

In the present study, in order to examine the role of mtDNA mutation in oral cavity cancers, we analyzed the MT-CYB and D-Loop sequences of the mitochondrial genome in 45 oral cavity cancers. We employed a sophisticated visualization technique, rainbow boxes (Lamy et al., 2016; Lamy et al., 2017), for identifying patients that share mutations at the same location and potential co-occurring mutations. This technique was recently proposed and applied to biological data, for example for designing a new schema of amino acids

${ }^{1}$ Department of Maxillofacial Surgery and Stomatology University Hospital Center Aristide le Dantec, ${ }^{2}$ GENGESPOP Team, Department of Animal Biology, Faculty of Science and Technology, Cheikh Anta Diop University, ${ }^{3}$ Department of Animal Biology, Faculty of Science and Technology, ${ }^{4}$ Cancer Institut, Faculty of Medicine, Pharmacy and Stomatology, Cheikh Anta Diop University, Dakar, Senegal, 'LIMICS, Université Paris 13, Sorbonne Paris Cité, 93017 Bobigny, France, INSERM UMRS 1142, UPMC Université Paris 6, Sorbonne Université, Paris, ${ }^{6}$ Laboratoire de Recherche en Informatique (LRI), CNRS, Université ParisSud, Université Paris-Saclay, 91405 Orsay, France. *For Correspondence: fatimata.mbaye@ucad.edu.sn 
(Lamy, 2018).

\section{Materials and Methods}

\section{Tissue samples}

Tumor tissue samples were derived from patients with oral cavity cancers. 45 patients treated in the Department of Oral and Maxillofacial Surgery at the Affiliated Hospital Aristide Le Dantec (Dakar, Senegal) were recruited for participation in this study. The study was approved by the University Cheikh Anta Diop Research Ethics committee (Reference: 0272/2018/CER/UCAD). The Helsinki Declaration has been read and approved. All cases were histologically confirmed and gave written informed consent for participation before surgery. As controls, peripheral blood samples $(\mathrm{N}=21)$ were collected from healthy individuals into EDTA-treated tubes.

\section{DNA extraction}

Genomic DNA was extracted using the QuickDNATM Miniprep Plus Kit (Zymo Research, Irvine, CA, USA) according to the manufacturer's instructions.

\section{MT-CYB PCR}

To determine the presence of mutations in the MT-CYB gene (encoding the cytochrome $\mathrm{b}$ protein), amplification of MT-CYB was performed in a $50-\mu 1$ reaction using $2 \mu 1$ of genomic DNA, $2.5 \mu 1$ of each primer, $5 \mu$ l buffer $10 \times$,

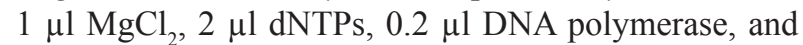
$34.8 \mu \mathrm{l}$ DNAse-free water. The PCR conditions were as follows: initial denaturation at $94^{\circ} \mathrm{C}$ for $3 \mathrm{~min} ; 40$ cycles of denaturation at $94^{\circ} \mathrm{C}$ for $45 \mathrm{~s}$, annealing at $52^{\circ} \mathrm{C}$ for $1 \mathrm{~min}$, and elongation at $72^{\circ} \mathrm{C}$ for $1 \mathrm{~min} 30 \mathrm{~s}$; followed by extension at $72^{\circ} \mathrm{C}$ for $10 \mathrm{~min}$ and holding at $10^{\circ} \mathrm{C}$. Primers H15915 (TCCCATTTCTGGTTTACAAGAC) and L14723 (ACCAATGACATGAAAAATCATGGTT) were used.

\section{D-Loop PCR}

Amplification of the D-Loop region from tissue and blood extracts was performed in a $50-\mu 1$ reaction using $2 \mu \mathrm{l}$ of genomic DNA, $2.5 \mu \mathrm{l}$ of each primer, 5 $\mu 1$ buffer $10 \times, 1 \mu 1 \mathrm{MgCl} 2,2 \mu 1 \mathrm{dNTPs}, 0.1 \mu \mathrm{l}$ DNA polymerase, and $34.9 \mu 1$ DNAase-free water. The following program was used initial denaturation at $95^{\circ} \mathrm{C}$ for $15 \mathrm{~min} ; 35$ cycles of denaturation at $95^{\circ} \mathrm{C}$ for $30 \mathrm{~s}$, annealing at $62^{\circ} \mathrm{C}$ for $30 \mathrm{~s}$, and elongation at $72^{\circ} \mathrm{C}$ for 2 $\mathrm{min}$; followed by extension at $72^{\circ} \mathrm{C}$ for $10 \mathrm{~min}$. Primers H408 (TGTTAAAAGTGCATACCGCCA) and L16340 (AGCCATTTACCGTACATAGCACA) were used.

\section{DNA sequencing}

All PCR products were purified and sequenced with an ABI Big Dye Terminator cycle sequencing ready reaction kit and an ABIPRISM 3730xl sequencer (Applied Biosystems, Foster City, CA, USA).

\section{Mutation visualization}

Sequences obtained from control and cancerous tissues were aligned using BioEdit software version 7.1.9 (Hall, 1999). In our analysis, each sample could present several mutations, and a given mutation could occur in several samples. Under these conditions, it can be difficult to determine which mutations are shared across samples and to identify possible co-occurring mutations. We used a visualization method based on rainbow boxes (Lamy et al., 2016; Lamy et al., 2017), a recently developed visualization technique for overlapping data sets. Rainbow boxes can be used to visualize up to 100 elements and 250 sets; we applied this technique to the visualization of the set of mutations present in each sample. Here, we considered samples as the elements and mutation locations as the sets. All mutations occurring at the same location were grouped together, for the purpose of visualization, but distinguished by color. Colors corresponded to mutation types for gene sequences and to predicted pathogenicity for protein sequences.

In rainbow boxes, elements (i.e., samples) are presented as columns, and sets (i.e., mutation locations) as rectangular boxes. Each box covers the columns corresponding to the samples that have a mutation at a given location. Whenever the samples carrying a given mutation are not displayed in contiguous columns, a "hole" is present in the box, represented by a thin link that relates the various parts of the box. Samples are ordered in the visualization so as to minimize the number of holes using Artificial Feeding Birds (AFB), a metaheuristic inspired by the behavior of pigeons (Lamy, 2019). As a consequence, similar samples are placed next to each other.

\section{Criteria for defining pathogenic mutations}

The D-Loop and MT-CYB regions of mtDNA derived from control and tumor tissues were analyzed, and any DNA sequence differences were regarded as somatic mutations. For MT-CYB, we used the following four in-silico algorithms to predict the putative effect of each nonsynonymous mutation on the cytochrome $\mathrm{b}$ protein function: Polyphen-2 (Adzhubei et al., 2013), SIFT (Kumar et al., 2009), mutationAssessor (mutationassessor. org), and Provean (Choi et al., 2012). A mutation was considered pathogenic when at least three methods predicted a deleterious effect. Mutation load was calculated as the percentage of nucleotides altered, considering only coding nonsynonymous mtDNA mutations.

\section{Results}

\section{Study population}

Using PCR and DNA sequencing, we investigated the occurrence of somatic point mutations in both the MT-CYB and D-Loop regions of the mitochondrial genome in oral cavity cancers from 45 patients and 21 controls. Patient age ranged from 14 to 86 . There were 17 male and 28 female patients. Patients were asked their smoking status and were classified according to their responses (Table 1).

\section{Visualization of mutations using rainbow boxes}

Figure 1 shows the mutations observed in MT-CYB and the D-Loop region and shared by oral cavity cancer tissues and controls, using rainbow boxes. Samples are shown in columns and grouped by health status (healthy control or oral cavity cancer patient). Samples are ordered 


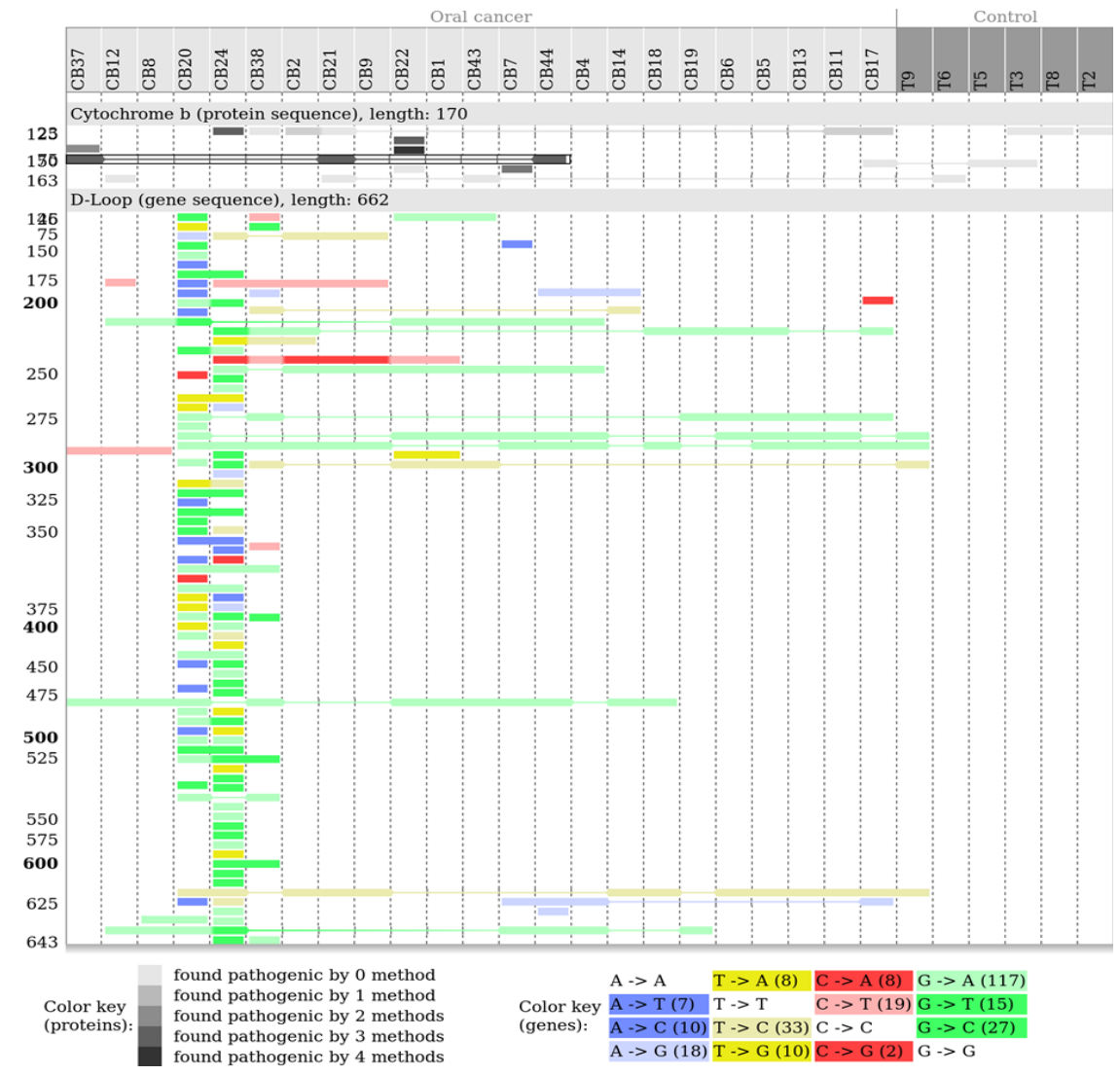

Figure 1. The Various Locations of the Mutations Observed in the Control and Oral Cavity Cancers Patients, Displayed as Rainbow Boxes.

by similarity, i.e. samples presenting similar mutations tend to be placed close to each other. Mutations are represented by rectangular boxes, with each box covering the columns of samples having the mutation. Boxes are organized vertically by sequence region (MT-CYB and D-Loop) and by mutation location. Different substitutions

Table 1. Patient Characteristics

\begin{tabular}{lc}
\hline Variable & Case $(\mathrm{N}=45)$ \\
\hline Sex & $17(37.77 \%)$ \\
Male & $28(62.22 \%)$ \\
Female & \\
Age & 16.64 \\
Std $+/-$ & 53.3 \\
Mean & $14-86$ \\
Range & \\
Smoking & $4(8.88 \%)$ \\
Tobacco Smoking & $9(20 \%)$ \\
Smoking + Alcohol & $1(2.22 \%)$ \\
Tobacco + Indian hemp + alcohol & $31(68.88 \%)$ \\
Never & \\
Localization & $16(37.20 \%)$ \\
Right & $23(53.48 \%)$ \\
Left & $1(2.32 \%)$ \\
Lower gum & $1(2.32 \%)$ \\
Mandibular gingiva & $2(4.65 \%)$ \\
Tongue &
\end{tabular}

at the same location, e.g. C242T and C242A, are grouped together in the same box but represented with different colors. For mutations in the D-Loop, we used colors to indicate the mutated base. The color hue is determined by the original base in the reference sequence, using the usual colors (A: blue, T: yellow, C: red, G: green). Bright colors correspond to transversions, and pastel colors to transitions. The color key also gives the number of mutations observed for each type.

For mutations in MT-CYB, we used a grayscale gradient to represent the predicted pathogenicity of each mutation. Darker boxes correspond to more pathogenic mutations, according to four in-silico methods detailed in the Materials and Methods section. The exact location and details of the various mutations can be obtained in popup bubbles.

In Figure 1, we can see that most mutations occurred in the D-Loop, and many of these were G-to-A transitions. Three patients (labeled CB24, CB10, and CB20) had a very high number of mutations in the second half of the D-Loop, with a high proportion of transversions, compared with mutation patterns observed in other patients. In addition, one patient (CB31) had many transversions in MT-CYB.

Somatic mtDNA mutation spectrum of Senegalese oral cavity cancer patients

The sequencing results for the tumor samples were compared with those obtained from controls. The distributions of mtDNA haplotypes differed between control and cancerous tissues [MT-CYB (control h=7; 
Table 2. Functional Impact of Amino Acid Substitutions in Cytochrome b Protein Identified in Patients with Oral Cavity Cancer

\begin{tabular}{|c|c|c|c|c|c|c|c|}
\hline \multirow[t]{2}{*}{ Mutation } & \multirow[t]{2}{*}{ rRCS position } & \multicolumn{4}{|c|}{ Functional impact } & \multirow{2}{*}{$\begin{array}{l}\text { Codon } \\
\text { changes }\end{array}$} & \multirow[t]{2}{*}{ Patients } \\
\hline & & MutationAssessor & Polyphen-2 & SIFT & Provean & & \\
\hline p.Leu52Pro & $\mathrm{L} 258 \mathrm{P}$ & neut & pod & - & neut & $\mathrm{CTA} \rightarrow \mathrm{CCA}$ & $\begin{array}{l}\text { CB2, CB11, CB17, } \\
\text { CB25, CB27, CB41 }\end{array}$ \\
\hline p.Leu52Gln & L258Q & neut & benign & - & neut & $\mathrm{CTA} \rightarrow \mathrm{CAA}$ & CB35 \\
\hline p.Leu52Arg & L258R & neut & benign & apf & neut & $\mathrm{CTA} \rightarrow \mathrm{CGA}$ & CB21 \\
\hline p.Ala53Asp & A259D & high & $\mathrm{pd}$ & - & del & $\mathrm{GCC} \rightarrow \mathrm{GAC}$ & CB22 \\
\hline p.Ala53Ser & $A 259 S$ & high & $\mathrm{pd}$ & apf & del & $\mathrm{GCC} \rightarrow \mathrm{TCC}$ & CB39 \\
\hline p.Asn54Ser & $\mathrm{N} 260 \mathrm{~S}$ & med & $\mathrm{pd}$ & apf & del & $\mathrm{AAC} \rightarrow \mathrm{AGC}$ & $\mathrm{CB} 22, \mathrm{CB} 35$ \\
\hline p.Asn57His & $\mathrm{N} 263 \mathrm{H}$ & low & pod & - & neut & $\mathrm{AAC} \rightarrow \mathrm{CAC}$ & CB31 \\
\hline p.Asn57Thr & $\mathrm{N} 263 \mathrm{~T}$ & neut & benign & tol & neut & $\mathrm{AAC} \rightarrow \mathrm{ACC}$ & CB38 \\
\hline p.Thr58Ile & $T 264 I$ & high & $\mathrm{pd}$ & apf & del & $\mathrm{ACC} \rightarrow \mathrm{ATC}$ & CB31 \\
\hline p.Pro60Thr & $P 266 T$ & low & $\mathrm{pd}$ & apf & del & $\mathrm{CCC} \rightarrow \mathrm{ACC}$ & CB31 \\
\hline p.His61Pro & $H 267 P$ & high & $\mathrm{pd}$ & apf & del & $\mathrm{CAC} \rightarrow \mathrm{CCC}$ & CB31 \\
\hline p.Pro64Arg & $P 270 R$ & high & $\mathrm{pd}$ & apf & del & $\mathrm{CCC} \rightarrow \mathrm{CGC}$ & CB39 \\
\hline p.Glu65Gly & $E 271 G$ & high & $\mathrm{pd}$ & - & del & $\mathrm{GAA} \rightarrow \mathrm{GGA}$ & $\mathrm{CB} 27$ \\
\hline p.Glu65Asp & $E 271 D$ & high & $\mathrm{pd}$ & apf & neut & $\mathrm{GAA} \rightarrow \mathrm{GAT}$ & CB24, CB31 \\
\hline p.Trp66Leu & $W 272 L$ & high & $\mathrm{pd}$ & apf & del & $\mathrm{TGA} \rightarrow \mathrm{TTA}$ & CB31 \\
\hline p.Leu69Val & $L 275 \mathrm{~V}$ & high & pd & apf & del & $\mathrm{CTA} \rightarrow \mathrm{GTA}$ & CB31 \\
\hline p.Phe70Leu & $F 276 L$ & med & $\mathrm{pd}$ & apf & del & $\mathrm{TTC} \rightarrow \mathrm{CTT}$ & CB31 \\
\hline p.Phe70Trp & F276W & - & $\mathrm{pd}$ & - & del & $\mathrm{TTC} \rightarrow \mathrm{TGG}$ & $\mathrm{CB}$ \\
\hline p.Phe70Val & $F 276 \mathrm{~V}$ & high & $\mathrm{pd}$ & - & del & $\mathrm{TTC} \rightarrow \mathrm{GTC}$ & CB27 \\
\hline p.Phe73Ser & $\mathrm{T} 279 \mathrm{~S}$ & neut & benign & apf & neut & $\mathrm{ACA} \rightarrow \mathrm{TCA}$ & CB31 \\
\hline p.Arg76Ala & $\mathrm{R} 282 \mathrm{~A}$ & - & pd & apf & del & $\mathrm{CGA} \rightarrow \mathrm{GCA}$ & CB39 \\
\hline p.Arg76Pro & $R 282 P$ & high & $\mathrm{pd}$ & - & del & $\mathrm{CGA} \rightarrow \mathrm{GCA}$ & $\begin{array}{c}\mathrm{CB} 21, \mathrm{CB} 32, \mathrm{CB} 33, \\
\mathrm{CB} 34, \mathrm{CB} 36, \mathrm{CB} 37, \\
\text { CB44 }\end{array}$ \\
\hline p.Val78Leu & V284L & med & benign & apf & neut & $\mathrm{GTC} \rightarrow \mathrm{CTC}$ & CB31 \\
\hline p.Leu82Met & L288M & - & $\mathrm{pd}$ & apf & neut & $\mathrm{CTA} \rightarrow \mathrm{ATA}$ & CB27 \\
\hline p.Leu86His & $L 292 H$ & high & $\mathrm{pd}$ & apf & del & $\mathrm{CTT} \rightarrow \mathrm{CAT}$ & CB27 \\
\hline p.Ser117Leu & S323L & neut & benign & tol & neut & $\mathrm{TCA} \rightarrow \mathrm{TTA}$ & CB22 \\
\hline p.Leu118F & L324F & low & pod & apf & del & $\mathrm{CTT} \rightarrow \mathrm{TTT}$ & CB7 \\
\hline p.Thr162Ile & T368I & neut & benign & tol & neut & $\mathrm{ACT} \rightarrow \mathrm{ATT}$ & $\begin{array}{c}\text { CB21, CB42, CB43, } \\
\text { CB45 }\end{array}$ \\
\hline p.Ile163Val & $\mathrm{I} 369 \mathrm{~V}$ & neu & benign & Tol & neut & $\mathrm{ATC} \rightarrow \mathrm{GTC}$ & $\mathrm{CB} 12$ \\
\hline
\end{tabular}

Pathogenic mutations are shown in bold; pathogenic mutations not previously described in mitomap database (www.mitomap.org) are shown in bold and italic; neut: neutral; tol, tolerable; pd, probably damaging; del, deleterious; pod, possibly damaging; apf, affect protein function; med, medium; ben, benign

cancerous h=20); D-Loop (control h=2; cancerous $\mathrm{h}=23$ )]. The term "haplotype" can also refer to the inheritance of a cluster of single nucleotide polymorphisms (SNPs), which are variations at single positions in the DNA sequence among individuals. Overall, 39 of the tumor tissues $(88.63 \%)$ had one or more somatic mtDNA mutations (11.4\% had one mutation, $0 \%$ tissues had two mutations, and $59.1 \%$ had at least three mutations). Twelve $(27.3 \%)$ patients had mutations in MT-CYB only, $18(40.9 \%)$ had mutations in the D-Loop only, and 9 $(20.5 \%)$ had mutations in both MT-CYB and the D-Loop. In total, $79.43 \%(309 / 389)$ of the somatic mutations in oral cavity cancer cases occurred in the D-Loop region of the mitochondrial genome, whereas $20.56 \%$ (80/389) occurred in the MT-CYB gene. In the D-Loop region, many more cancer patients than controls carried multiple single-nucleotide polymorphisms (Figure 1). Frequent mutations were reported in oral cavity cancers at positions G229A (8 patients), G232A (10 patients), G245A (10 patients), $G 275 A$ (10 patients), G277A (15 patients), G281A (15 patients), G641A (7 patients), G477A (14 patients), and $T 606 C$ (16 patients). The relative mutation frequency (mutations/nucleotides) for the D-Loop was 2.99-fold higher than that for MT-CYB (309/662 versus $80 / 513)$. The most frequent mutation types were base substitutions: G-to-A (149) or C-to-T (25) transition 
mutations $(73.10 \%, 174 / 238)$, followed by A-to-G (21) and T-to-C (43) transitions.

\section{Predicted pathogenicity of missense mutations}

Mutations in MT-CYB were identified in $27.3 \%$ of oral cavity cancer tissues, with 29 missense mutations reported (Table 2). Among the mutations found, 16 (55.17\%), including p.Ala53Asp, p.Ala53Ser, p.Asn54Ser, p.Thr58Ile, p.Pro60Thr, p.His61Pro, p.Pro64Arg, p.Glu65Gly, p.Glu65Asp, p.Trp66Leu, p.Leu69Val, p.Phe70Leu, p.Phe70Val, p.Arg76Ala, p.Arg76Pro, and p.Leu86His, were considered pathogenic. These amino acid changes were induced by 32 mutations, including $9(28.12 \%)$ transitions and $23(71.87 \%)$ transversions. The most common pathogenic mutation ( 7 patients) was an arginine to proline substitution at position 76 (p.Arg76Pro). This mutation is novel and has not been previously reported in the database MITOMAP (www. mitomap.org). The effect of somatic mtDNA mutations in $M T-C Y B$ was evaluated in terms of the mutation load. The pathogenic mutation load was 3.11\% (16/513), and pathogenic mutations were carried by $11(25 \%)$ patients.

\section{Discussion}

In this study, we present for the first time a comprehensive mutation screen of selected regions of the mitochondrial genome in oral cavity cancers in Senegalese patients. Among the oral cavity tumor tissues, $88.63 \%$ carried mitochondrial mutations. The frequency of mutation was much higher in the non-coding D-Loop region $(79.43 \%)$ than in $M T-C Y B(20.56 \%)$, with a relative mutation frequency 2.99-fold higher than that in $M T-C Y B$. Other studies report a 7-fold increase in susceptibility (Zhu et al., 2005). The D-Loop region of mtDNA is crucial for replication and expression of the mitochondrial genome, as it contains essential transcriptional promoters and the leading-strand origin of replication. In contrast to the findings of the present study, a study by Lin et al., (2015) involving direct sequencing reported somatic mutations in the D-Loop in $62.5 \%$ of oral squamous cell carcinoma patients. Tan et al., (2004), in their study of betel quid-related oral cancer patients, observed somatic mutations in the D-Loop region in $66.7 \%(12 / 18)$ of samples (Tan et al., 2004). A recent study on oral squamous cell carcinoma also found a high rate of somatic mutations in the D-Loop region, with mutations in 85\% (203/240) of patients (Lai et al., 2013). The high somatic mutation rates observed in the aforementioned studies are comparable with the rate found in our study.

Oral cavity cancers in Senegalese patients revealed high intratumoral heterogeneity. Twelve patients who had mutations in $M T-C Y B$ did not have mutations in the D-LoopMT-CYB mutations do not appear to be associated with those of the D-Loop. Cells in the same tumor are therefore not identical. This intratumoral heterogeneity can be a limiting factor in determining appropriate treatment. For example, it is unclear which of the two mitochondrial regions (MT-CYB or the D-Loop) is associated with tumorigenesis, including changes in the cell cycle, apoptosis, and differentiation. It is also unknown which of the mutations in the two regions are responsible for disease dissemination.

The distribution of mutation types may provide some insight into the mechanisms that cause these mutations. The majority of the base substitution mutations were either G-to-A (149) or C-to-T (25) transitions, a spectrum characteristic of oxidative DNA damage (Chatterjee et al., 2006). To investigate the possibility that $M T-C Y B$ and D-Loop mitochondrial sequences may be associated with cancer development, we assessed the relative frequency of mutation in each region.

Human mtDNA sequences are highly variable. It is therefore necessary to define functionally important somatic mutations that may be deleterious or pathogenic in terms of tumor progression. A key step in the process of annotating sequencing results is the integration of pathogenicity predictions. This encompasses the use of various tools that aim to predict the effects of substitutions on the structure and/or function of proteins. Several tools are available and use different approaches. Although these methods are useful in practice, their accuracy remains a concern. Therefore, we applied a battery of four tools to determine the pathogenicity of mutations, as described in the Materials and Methods section, and we found that $11(25 \%)$ oral cavity cancers carried nonsynonymous pathogenic mutations. This is similar to the results of another study showing that $23.6 \%$ of oral cavity squamous cell carcinoma cases derived from a Taiwanese population carried pathogenic mutations in mtDNA sequences (Lai et al., 2013). The cytochrome b protein plays a key role in mitochondria, which convert the energy from food into a form that cells can use. Mutations in the MT-CYB gene can cause mitochondrial complex III deficiency.

Many cancer studies completely ignore synonymous mutations (Sankin et al., 2014), whereas others use them to build neutral background models of mutation clustering for the detection of activating mutations (Tamberero et al., 2013). Apart from their silent effect on the encoded protein, the disregard for such DNA modifications may be rooted in the assumption that synonymous sites are not subject to selection (Kimura, 1977). However, there are many examples of synonymous mutations that have been implicated in various human diseases, including heritable conditions (Sauna and Kimchi Sarfaty, 2011), suggesting parallel functional effects in cancers. Awareness that somatic synonymous mutations may include causal variants, i.e. driver mutations, is beginning to take hold. In this study, the mutations in the gene encoding cytochrome $\mathrm{b}$ follow the neutral theory of molecular evolution. Most mutations are selectively neutral and would have occurred at random. The $M T-C Y B$ mutations are somatic, and the majority are synonymous substitutions.

We used a new method for visualizing mutations shared by patients, based on rainbow boxes. This is the first application of rainbow boxes in this context. This type of visualization was found to be useful for searching for mutation co-occurrence and could be used on many other datasets.

The discovery of cancer-specific mitochondrial DNA mutations has escalated within recent years. Our findings may provide further evidence for the theory that most 
oral cavity tumors contain mitochondrial mutations. Future studies should aim to establish the actual causes of intratumoral heterogeneity and the correlation between oral cavity cancers and risk factors (e.g. sex, age, and smoking status).

\section{Conflict of interest}

The authors declare no conflict of interest.

\section{Acknowledgements}

The authors are grateful to Department of Oral and Maxillofacial Surgery at the Affiliated Hospital Aristide Le Dantec for the availability of the samples. The study was approved by the University Cheikh Anta Diop Research Ethics committee (Reference: 0272/2018/CER/UCAD). The Helsinki Declaration has been read and approved. This study was funded by the Population Genetics and Management Team of the Department of Animal Biology of the Faculty of Science and Technology, Cheikh Anta Diop University, Dakar.

\section{References}

Adzhubei I, Jordan DM, Sunyaev SR (2013). Predicting functional effect of human missense mutations using polyPhen-2. Curr Protoc Hum Genet, 7, Unit 7.20.

Chatterjee A, Mambo E, Sidransky D (2006). Mitochondrial DNA mutations in human cancer. Oncogene, 25, 4663-74.

Chaturvedi AK, Andersonn WF, Lartet-Tieulent J, et al (2013). Worldwide trends in incidence rates for oral cavity and oropharyngeal cancers. J Clin Oncol, 31, 4550-9.

Choi Y, Sims GE, Murphy S, Miller JR, Chan AP (2012). Predicting the functional effect of amino acid substitutions and indels. PLoS One, 7, e46688.

Hall TA (1999). BioEdit: a user-friendly biological sequence alignment editor and analysis program for Windows 95/98/ NT. Nucleic Acids Symp Ser, 44, 211-32.

Ishikawa K, Takenaga K, Akimoto M, et al (2008). ROS-generating mitochondrial DNA mutations can regulate tumor cell metastasis. Science, 320, 661-4.

Jakupciak JP, Wang W, Markowitz ME, et al (2005). Mitochondrial DNA as a cancer biomarker. J Mol Diagn, 7, 258-67.

Kimura M (1977). Preponderance of synonymous changes as evidence for the neutral theory of molecular evolution. Nature, 267, 275-6.

Kumar P, Henikoff S, Ng PC (2009). Predicting the effects of coding non-synonymous variants on protein function using the SIFT algorithm. Nat Protoc, 4, 1073-81.

Lai CH, Huang SF, Liao CT, et al (2013). Clinical significance in oral cavity squamous cell carcinoma of pathogenic somatic mitochondrial mutations. PLoS One, 8, e65578.

Lamy JB, Berthelot H, Favre M (2016). Rainbow boxes: a technique for visualizing overlapping sets and an application to the comparison of drugs properties. International Conference on Information Visualisation (iV), pp 253-60.

Lamy JB, Berthelot H, Capron C, Favre M (2017). Rainbow boxes: a new technique for overlapping set visualization and two applications in the biomedical domain. J Visual Lang Comput, 43, 71-82.

Lamy JB (2018). A new diagram for amino acids: User study comparing rainbow boxes to Venn/Euler diagram. International Conference on Information Visualisation (iV).

Lamy JB (2019). Artificial Feeding Birds (AFB): a new metaheuristic inspired by the behavior of pigeons. In:
Advances in nature-inspired computing and applications (Shandilya SK, Shandilya S, Nagar AK, editors). Berlin, Springer, pp 43-60.

Lin JC, Wang CC, Jiang RS, Wang WY, Liu SA (2015). Impact of somatic mutations in the D-Loop of mitochondrial DNA on the survival of oral squamous cell carcinoma patients. PLoS One, 10, e0124322.

Petros JA, Baumann AK, Ruiz-Pesini E, et al (2005). mtDNA mutations increase tumorigenicity in prostate cancer. Proc Natl Acad Sci U S A, 102, 719-24.

Sankin A, Hakimi AA, Mikkilineni N, et al (2014). The impact of genetic heterogeneity on biomaker development in Kidney cancer assessed by multiregional sampling. Cancer Med, 3, 1485-92.

Sauna ZE, Kimchi-Sarfaty C (2011). Understanding the contribution of synonymous mutations to human disease. Nat Rev Genet, 12, 683-91.

Stewart JB, Freyer C, Elson JL, Larsson NG (2008). Purifying selection of mtDNA and its implications for understanding evolution and mitochondrial disease. Nat Rev Genet, 9, 657-62.

Tamberero D, Gonzalez-Perez A, Lopez-Bigas N (2013). Oncodrive Clust: exploiting the positional clustering of somatic mutations to identify cancer genes. Bioinformatics, 29, 2238-44.

Tan DJ, Chang J, Chen WL, et al (2004). Somatic mitochondrial DNA mutations in oral cancer of betel quid chewers. Ann NY Acad Sci, 1011, 310-16.

Wamakulasuriya S (2009). Global epidemiology of oral and oropharyngeal cancer. Oral Oncol, 45, 309-16.

Zhu W, Qin W, Bradley P, et al (2005). Mitochondrial DNA mutations in breast cancer tissue and in matched nipple aspirate fluid. Carcinogenesis, 26, 145-52.

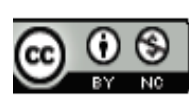

This work is licensed under a Creative Commons AttributionNon Commercial 4.0 International License. 